\title{
Accurate and Energy Efficient ECG Analysis Method for ECG Monitoring System
}

\author{
Min Zeng ${ }^{*}$, Regular Member Jeong-Gun Lee ${ }^{* *}$ Il-Yong Chung ${ }^{* * *}$, Jeong-A Lee ${ }^{* * * * \circ}$ Lifelong Member
}

\section{ABSTRACT}

This paper proposes an energy efficient ECG monitoring system by putting some intelligence on the sensor node to reduce the number of transmissions. The sensor node is mostly put into the processing mode and just connects the base station when necessary. Therefore, the transmission energy is greatly reduced while the energy for processing is increased a little bit. Our proposed ECG analysis method classifies ECG cycles by computing the Euclidean distance between the sensed ECG cycle and the reference ECG cycle. This work is a detailed and full explanation of our former work. Extended experimental results show that the proposed trade is very effective in saving energy and the Euclidean distance based classification method is accurate. Furthermore, the PowerTOSSIM energy simulation method is also demonstrated as very accurate in evaluating the energy consumption of the sensor node in our application scenario.

Key Words : Body sensor network, ECG analysis, Energy efficiency, ECG monitoring, PowerTOSSIM.

\section{I . Introduction}

Body Sensor Network (BSN) based ECG monitoring system has great potential for managing people's chronic health conditions. Nowadays, most of the ECG monitoring schemes and projects aim at long term monitoring while the sensor nodes have to detect and transmit the ECG signal to the base station continuously. This makes the battery powered sensor node quickly run out of power. The proposed low power ECG monitoring system in this paper puts some intelligence on a sensor node to reduce the number of transmission ${ }^{\mathrm{s}[1]}$. The sensor node is mostly put into the processing mode and just connects the base station when necessary.

The rest of this paper is structured as follows. Section 2 provides an overview of the existing human body monitoring systems and the recent advances in this field. Section 3 presents the proposed system architecture. Section 4 describes the local ECG analysis algorithm. Section 5 presents implementation, simulation and experimental results. Finally, Section 6 discusses conclusion and future work.

\section{Related Work}

As energy consumption is one of the most critical problems in BSN systems, many designs related with transceivers, processors, and platforms have been proposed to save power in ECG monitoring systems. $\operatorname{In}^{[2]}$, a low power, low cost, high integrated transmitter working at the $2.4 \mathrm{GHz}$ ISM band is presented. More aggressively, a $1 \mathrm{~V}$ $\mathrm{RF}$ transceiver for BSN applications is presented

※ This study was supported (in part) by research funds from Chosun University, 2011.

* Min Zeng is with Department of Computer Engineering, Chosun University, Gwangju, Korea (e-mail: rainyday_129@hotmail.com),

** Jeong-Gun Lee is with Department of Computer Engineering, Hallym University, Gangwon-do, Korea (email: Jeonggun.Lee@hallym.ac.kr), ***Il-Yong Chung is with Department of Computer Engineering, Chosun University, Gwangju, Korea (email: iyc@mail.chosun.ac.kr), ****Jeong-A Lee is with Department of Computer Engineering, Chosun University, Gwangju, Korea (e-mail: jalee@chosun.ac.kr), ( ${ }^{\circ}$ : Corresponding Author)

논문번호 : KICS2012-02-100, 접수일자 : 2012년 2월 29일, 최종논문접수일자 : 2012년 4월 30일 
$\mathrm{in}^{[3]}$. ECO sensor node, developed at University of California, is an ultra-wearable, low power, and expandable wireless sensor node ${ }^{[4]}$. Besides these new hardware designs, a number of protocols have also been proposed as being efficient in saving power. A novel Time Division Multiple Access (TDMA) MAC protocol called H-MAC is described $\mathrm{in}^{[5]}$. It improves BSN energy efficiency by exploiting heartbeat rhythm information to perform time synchronization. $\mathrm{In}^{[6]}$, an energy and mobility aware geographical multipath routing scheme for wireless sensor networks in a pervasive healthcare system is proposed, which can extend the network lifetime and reduce the packet loss rate and link failure rate.

Despite so many concerns about energy efficiency in BSN systems, a few studies have been published in the real-time system operation field for saving power. $\mathrm{In}^{[7]}$, the local ECG analysis method based on ECG features (R-R interval, QRS width, etc.) is proposed. Using the local ECG analysis algorithm on sensor node described in $^{[8]}$, the data packets overload and power consumption can both be reduced. This paper shows the detailed classification accuracy and power efficiency of using our real-time on-node processing method.

\section{System Architecture}

The proposed two-tier architecture comprises a number of base stations, each controlling several sensor motes with ECG analysis function. Base stations at the upper tier are assumed to be rich in computational, communication, and storage resources. The task of this tier is to gather data from the bottom tier and answer queries posed by the user. In contrast, the sensor nodes in the bottom tier are assumed to be low-power mote platforms. Sensor nodes in the proposed scheme attempt to do a preliminary ECG signal processing as ECG cycle analysis and only transmit the abnormal ECG cycle instead of transmitting every ECG cycle.

The sensor node performs the local ECG diagnosis function as follows: at each sampling time $t$, the sensed ECG data is stored in the EEPROM of the sensor node. After getting enough data, the local ECG analysis algorithm is run to locate one ECG cycle, and then model the current cycle. Finally the current cycle and the standard cycle are compared to determine whether or not it is necessary to give doctors and nurses an alarm signaling an abnormality of this patient's heart by launching a radio transmission. As soon as doctors and nurses receive this alarm through the base station, they can require the sensor node to transmit the recent ECG data stored in the sensor node and take urgent measures to help this patient.

In traditional continuous transmission schemes, sensor nodes continuously transmit ECG data to the base station with each packet containing 10 data. Hence, to successfully transmit one full ECG cycle, 30 transmission should be launched (each cycle consists of 300 ECG data and for the radio transmission one package is transmitted at one time). In contrast, sensor nodes in our system usually archive the sensed ECG data and transmit an alarm to the base station only when the node determines that the signal is abnormal through on-node ECG diagnosis. In the proposed system, the sensor node trades communication for computation. Since the processing mode is far more energy efficient than the radio transmission mode, much power can be saved in this way. Next we describe the local ECG analysis algorithm in detail.

\section{Local ECG Analysis Algorithm}

The local ECG analysis algorithm is a real-time ECG cycle detection and classification method on resource limited sensor nodes based on the autoregressive model and the calculation of an Euclidean distance. The local ECG analysis algorithm on the sensor node includes R-peak detection, autoregressive model and Euclidean distance.

Before modeling, the ECG signal is 
preprocessed to remove noises. Then the peak within three hundred samples is detected and it is considered as the $R$ peak. One cardiac cycle is captured as one hundred samples before the $\mathrm{R}$ peak and two hundred samples after the $\mathrm{R}$ peak. Currently it is adequate to capture most of the information from a particular cardiac cycle. Next the AR model is used to model the ECG signal and Burg's algorithm ${ }^{[9]}$ is used to calculate the AR coefficients. Compared with other complex models, this model is more suitable for our source limited system. AR models is given by

$$
v[k]=\sum_{i=2}^{P+1} a_{k} v[k-i+1]+n[k]
$$

Where $v[k]$ is the ECG time series, $n[k]$ is zero mean white noise, $a_{k}$ is the AR coefficients, and $P$ is the AR order. A very important issue in AR modeling is the AR order used to model a signal. A proper AR order is critical to model a signal with sufficient accuracy for classification. As mentioned in ${ }^{[10]}$, an AR order chosen as 4 is enough to model the ECG signal.

After preprocessing and modeling, classification techniques are necessary for the efficient classification of the cycle to cycle patterns into predefined output categories (normal, abnormal). Stage-by-stage classification or more complex methods for classifying various ECG diseases are not efficient enough to satisfy the light-weight requirement of resource limited sensor node. In our work, only a primary step of classification is performed considering the resource limitation of sensor nodes. The simple but effective classification method is adopted by computing the Euclidean distance between the AR coefficients from the tested ECG signal and the normal ECG signal of the same person. The Euclidean distance d between points $A=\left(a_{1}, a_{2}, \ldots, a_{n}\right)$ and $B=\left(b_{1}, b_{2}\right.$, $\left.\ldots, b_{n}\right)$ is given by

$$
d=\sqrt{\left(a_{1}-b_{1}\right)^{2}+\left(a_{2}-b_{2}\right)^{2}+\ldots+\left(a_{n}-b_{n}\right)^{2}}
$$

We choose Euclidean distance as the measure of similarity because a specific and complex classification method is not suitable for real-time operation for a resource limited sensor node. By calculating the Euclidean distance between a sensed ECG signal and a reference ECG signal, only those cycles that deviate significantly from the standard cycles are reported to the base station. The sensor node specifies a threshold $\delta$ that defines the worst-case deviation that can be tolerated. Let $d_{t}$ denote the actual calculated distance between real time cycle and the reference cycle of the same patient.

As indicated earlier, computation of $d_{t}$ using (2) involves reading of a series of real-time ECG data and a few floating point subtractions, multiplications and extractions, all of which are inexpensive when compared to the cost of the Radio's communication power.

\section{Evaluation}

This section presents the evaluation of the proposed ECG analysis algorithm from two aspects: validation of the simulated power consumption data against real mote, and power consumption comparison of different ECG monitoring schemes.

\subsection{Validation of Simulated Power Consumption}

ECG monitoring application programs are written and compiled in $\mathrm{NesC}^{[11]}$ on TinyOS ${ }^{[12]}$. Power consumption is estimated using PowerTOSSIM, which is a power modeling extension to TOSSIM simulator in TinyOS. The power consumption is measured for five components - CPU, RADIO, LED, SENSOR, EEPROM. To validate PowerTOSSIM's simulated power consumption, we run the ECG monitoring application both on PowerTOSSIM and on an actual mote. The ECG monitoring application used here is the continuous monitoring scheme, in which sensor mote continuously transmits sensed data to base station without ECG cycle 
processing. The real sensor node used is the Hybus mote ${ }^{[13]}$, which consists of a MSP430 processor, a $2.4 \mathrm{GHz} \mathrm{CC} 2420$ radio, and $512 \mathrm{kB}$ EEPROM. The sampling rate is $250 \mathrm{~Hz}$. Sensor nodes and base stations communicate wirelessly through the embedded antenna integrated in sensor nodes. X4-LIFE Inspector II is used to measure the power consumption of the real mote. The overhead of using this power meter is about $29996.49 \mathrm{~mJ}$ and numerical processing is done to remove this measuring overhead to obtain the power consumption of the sensor node.

Table 1 presents total simulated and measured energy consumption of continuous ECG monitoring application for various running time. As the table shows, PowerTOSSIM achieves excellent accuracy, with a near-zero average error compared to the real mote, and a maximum error of about $4.1 \%$. Some of this difference between simulated and measured energy can be attributed to instrumental error and noise. Other differences may be due to rounding errors in experimental setup.

Table 1. Total energy consumption: simulated energy vs. measured energy.

\begin{tabular}{cccc}
\hline \multirow{2}{*}{ Time(s) } & \multicolumn{3}{c}{ Total energy consumption $(\mathrm{mJ})$} \\
\cline { 2 - 4 } & Simulated & Measured & Error(\%) \\
\hline 60 & 2304.57 & 2403.51 & -4.1 \\
120 & 4706.87 & 4807.02 & -2.1 \\
180 & 7075.21 & 7210.52 & -1.9 \\
240 & 9580.77 & 9614.03 & -0.3 \\
300 & 12389.08 & 12017.54 & 3.1 \\
600 & 24679.76 & 24035.08 & 2.7 \\
900 & 37007.44 & 36052.62 & 2.6 \\
\hline
\end{tabular}

\subsection{Power Consumption Comparison}

Our experiments center on two issues: classification accuracy and power consumption. Here we focus on the power issue. For the accuracy issue, we adopt test data set from MIT-BIH malignant ventricular ectopy database, Sudden Cardiac death holter database, and QT database. NSR, APC, PVC, SVT, VT, and VF cycles are acquired from these databases for ECG modeling and classification. Fig. 1 shows the classification accuracy with different threshold $\delta$ in our ED based method. We find that $\delta=0.6$ achieves the best performance. The detailed classification accuracy of GLM based and the Euclidean Distance (ED) based methods are shown in Table $2^{[14]}$. Simulation results show that the ED based method is very efficient in distinguishing abnormal ECG cycles from normal cycles which is very important to our design.

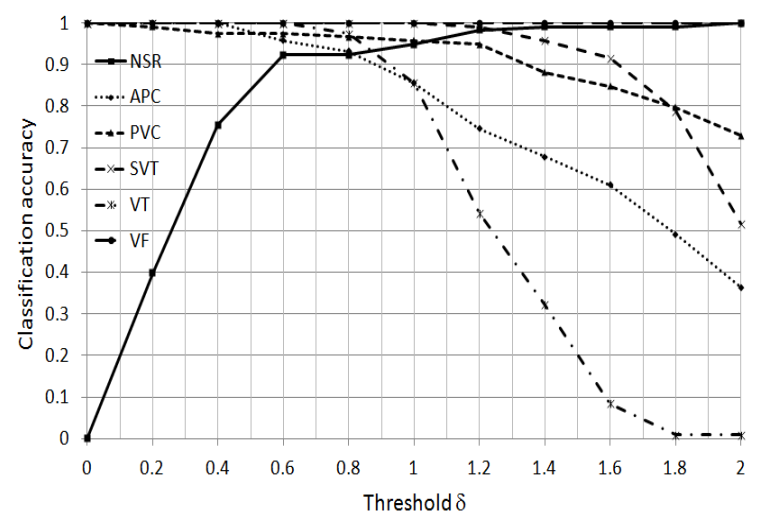

Fig. 1. Classification accuracy with different threshold.

Table 2. Classification accuracy comparison.

\begin{tabular}{cllllll}
\hline \multirow{2}{*}{$\begin{array}{c}\text { Meth } \\
\text { ods }\end{array}$} & \multicolumn{6}{c}{ Accuracy } \\
\cline { 2 - 7 } & NSR & APC & PVC & SVT & VT & VF \\
\hline GLM & $90 \%$ & $69 \%$ & $90 \%$ & $99 \%$ & $96 \%$ & $55 \%$ \\
ED & $92 \%$ & $96 \%$ & $97 \%$ & $100 \%$ & $100 \%$ & $100 \%$ \\
\hline
\end{tabular}

Next we present power evaluation results obtained by varying the simulation time. We study four cases for the sensor node: continuously transmit ECG signal to the base station with radio always on; continuously transmit ECG signal to the base station with radio on only when transmission is required; perform ECG analysis locally with every detected ECG cycle as abnormal cycle; perform ECG analysis locally with every detected ECG cycle as normal cycle.

The estimated energy consumption of the proposed system sensor node and the sensor node performing continuous transmission is shown in Fig. 2. The horizontal line in the figure denotes 


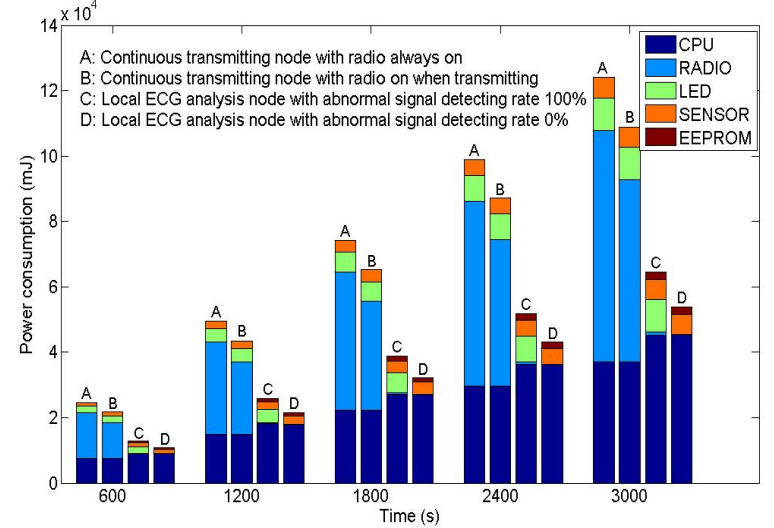

Fig. 2. Accumulated energy consumption: Local ECG analysis node vs. Continuous transmission node.

the total accumulated energy consumption of each simulation time with different schemes mentioned above. In the power consumption of traditional sensor node without local ECG analysis function, more than half of the total energy is used up by the radio component. In our proposed system, storing ECG data performed by EEPROM component and ECG analysis performed by CPU component slightly increases the energy consumption, while Radio transmission is not always necessary and accordingly the Radio component energy consumption is greatly reduced. Fig. 2 also shows that the longer the monitoring time, the more the energy can be saved.

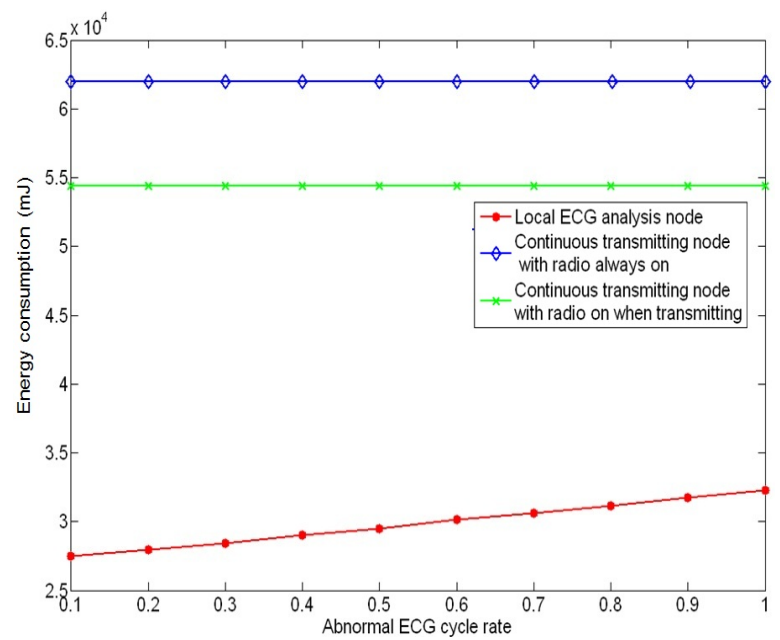

Fig. 3. Energy consumption for different abnormal ECG cycle rate.

Fig. 3 shows the abnormal ECG cycle rate associated with the total energy consumption of the sensor node which handles the local ECG analysis. With the increase of the abnormal ECG cycle rate, the total energy consumption also increases as radio should be turned on more frequently to report abnormality to the base station. Compared with the power consumption of continuous transmitting node, the superiority of the local ECG analysis node is obvious. In the continuous transmission node, e consumption does not vary with the abnormal ECG cycle rate and it almost doubles the energy consumed by the local ECG analysis node. If we assume that we use 2 AA batteries in series with a capacity of 2500 $\mathrm{mAh}$, the battery life time approximately becomes 7 days for the continuous transmission node, but more than half a month for the local ECG analysis node.

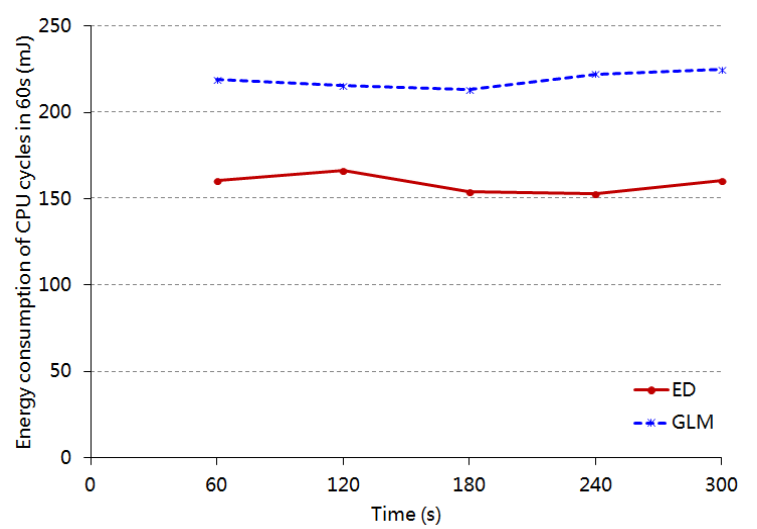

Fig. 4. Energy consumption of CPU cycles in 60 s.

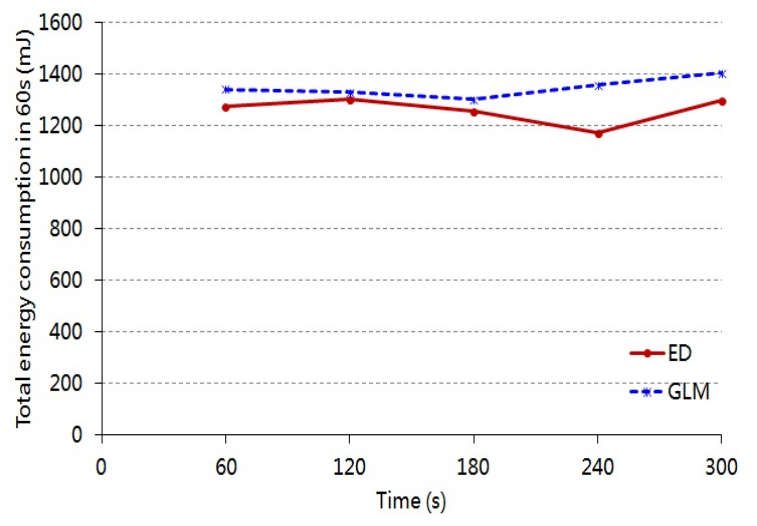

Fig. 5. Total Energy consumption in 60 s.

In Fig. 4, energy consumption of CPU cycles for ED and GLM based classification methods at 
intervals of $60 \mathrm{~s}$ is observed. Obviously, there is a certain gap of energy consumption between these two schemes and ED based method saves much more energy when compared with GLM based method. On average, the CPU cycle energy consumption in every $60 \mathrm{~s}$ of using ED based method is $158.84 \mathrm{~mJ}$, while it is $218.88 \mathrm{~mJ}$ of using GLM based method. Energy consumption in $60 \mathrm{~s}$ is reduced by up to $31.21 \%$ by using ED based classification method.

In Fig. 5, total energy consumption includes five parts: CPU, RADIO, LED SENSOR, and EEPROM. Experimental result shows that the total energy consumption difference of ED and GLM methods is smaller than that of the CPU cycles. Energy consumption of ED based method can be reduced at most $13.63 \%$ in 60 s when compared with GLM method.

This is due to the computation delay of GLM based method. Specifically, in each cycle, The GLM based method requires more time to finish ECG analysis. Therefore, the total number of ECG cycles that can be used for diagnosis is smaller than using ED based method in specific time duration, and accordingly the energy consumption of other components such as RADIO is smaller than using ED based method which leads to a decrease in total energy consumption. In other words, GLM based method results in smaller energy consumption gap with ED based method in a specific time interval, since a smaller number of ECG cycles are processed. This processing delay of GLM based method postpones the time of detecting any possible abnormal ECG cycles, which may lead to loss of the best treatment timing.

\section{Conclusions}

This paper described a two-tier low power ECG monitoring system. In contrast to existing techniques, our work puts some intelligence on an energy restricted sensor node in order to save power by trading transmission for computation. We design an ECG analysis algorithm on 408 embedded mote platforms and these platforms are always resource restricted. The novel aspect of our work is the use of autoregressive model to model the ECG signal and the Euclidean distance as a simplified preliminary classification method. Through a detailed four case study, we analyzed a continuous transmission scheme and local ECG analysis scheme for sensor nodes and showed that our proposed system is much more energy-efficient. Our experiments showed that this local ECG analysis algorithm yields an improvement in saving of about $39-57 \%$ energy of the sensor node and it prolongs the lifetime of the ECG monitoring system. As part of the future work, we plan to extend the sensor node with another flash memory board to archive a larger amount of historical data at an extremely low energy cost and a smaller delay.

\section{References}

[1] M. Zeng, I. Y. Chung, J. A. Lee, J. G. Lee, "An on-node intelligence based energy efficient ECG monitoring system," in Proc. of 2011 International Conference on ICT Convergence (ICTC), pp. 401-405, Sep. 2011.

[2] J. M. Gu, W. M. Lim, K. S. Yeo, M. A. Do, and C. C. Boon, "Low power transmitter design for BAN," in Proc. of Biomedical Circuits and Systems Conference, pp. 175 - 178, Nov. 2007.

[3] A. Wong, G. Kathiresan, T. Chan, O. Eljamaly, O. Omeni, D. McDonagh, A. Burdett, and C. Toumazou, "A 1V wireless transceiver for an ultra low power SoC for biotelemetry applications," Solid-State Circuits, vol. 43, no. 7, pp. 1511-1521, 2008.

[4] C. Park, P. H. Chou, "Eco: ultra-wearable and expandable wireless sensor platform," in Proc. of International Workshop on Wearable and Implantable Body Sensor Networks, pp. 162-165, Apr. 2006.

[5] H. M. Li, and J. D. Tan, "Medium access 
control for body sensor networks," in Proc. of 16th International Conference on Computer Communications and Networks, pp. 210-215, Aug. 2007.

[6] X. L. Wu B. J. d'Autriol, J. S. Cho, S. Y. Lee, "Optimal routing in sensor networks for in-home health monitoring with multi-factor considerations," in Proc. of 6th Annual IEEE International Conference on Pervasive Computing and Communications, pp. 720-725, Mar. 2008.

[7] D. Lee, S. Bhardwaj, E. Alasaarela, and W. Chung, "An ECG analysis on sensor node for reducing traffic overload in $\mathrm{u}$-healthcare with wireless sensor network," in Proc. of 6th IEEE Sensors Conference, pp. 256-259, Oct. 2007.

[8] M. Zeng, J. G. Lee, G. S. Choi, and J. A. Lee, "Intelligent sensor node based a low power ECG monitoring system," IEICE Electron Express, vol. 6, no. 9, pp. 560-565, 2009.

[9] J. P. Burg, "Maximum entropy spectral analysis," PhD dissertation, Department of Geophysics, Stanford University, May. 1975.

[10] D. F. Ge, N. Srinivasan, and S. M. Krishnan, "Cardiac arrhythmia classification using autoregressive modeling," Biomedical Engineering Online, vol. 1, no. 5, pp. 1-12, 2002.

[11] D. Gay, P. Levis, and R.von Behren, "The nesC language: a holistic approach to networked embedded systems," in Proc. of the ACM SIGPLAN conference on Programming language design and implementation, Jun. 2003.

[12] J. Hill, R. Szewczyk, A. Woo, S. Hollar, D. Culler, and K. Pister, "System architecture directions for networked sensors," in Proc. of 9th International Conference on Architectural Support for Programming Languages and Operating Systems, pp. 93-104, 2000.

[13] Hybus mote. www.hybus.net

[14] M. Zeng, and J. A. Lee, "Power efficient classification method for sensor nodes in BSN based ECG monitoring system," The Journal of Korea Information and Communication Society, vol. 35, no. 9, pp. 1322-1329, 2010.

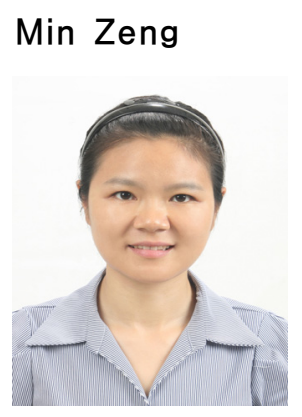

Min Zeng is a Ph.D. student in the Department of Computer Engineering at Chosun University. Her research interests include low power architectures and protocols on body sensor networks.

\section{Jeong-Gun Lee}

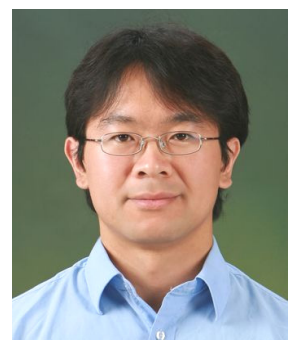

Jeong-Gun Lee is an assistant professor at the department of computer engineering, Hallym University. His research interests include Multi-core design, reconfigurable system design.

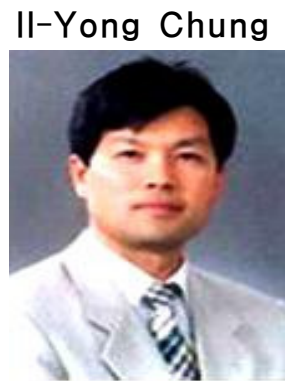

Il-yong Chung is a Professor in Department of Computer Science, Gwangju, Korea. His research interests are in computer networking, security systems and coding theory.

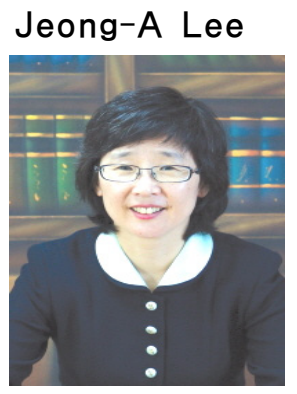

Jeong-A Lee is presently a Professor of Department of Computer Engineering, Chosun University. Her research interests include computer architecture, fast digital and CORDIC arithmetic, and configurable computing. 